\title{
Multiple Mechanosensory Modalities Influence Development of Auditory Function
}

\author{
Seth S. Horowitz, Leslie H. Tanyu, and Andrea Megela Simmons \\ Departments of Psychology and Neuroscience, Brown University, Providence, Rhode Island 02912
}

\begin{abstract}
Sensory development can be dependent on input from multiple modalities. During metamorphic development, ranid frogs exhibit rapid reorganization of pathways mediating auditory, vestibular, and lateral line modalities as the animal transforms from an aquatic to an amphibious form. Here we show that neural sensitivity to the underwater particle motion component of sound follows a different developmental trajectory than that of the pressure component. Throughout larval stages, cells in the medial vestibular nucleus show best frequencies to particle motion in the range from 15 to $65 \mathrm{~Hz}$, with displacement thresholds of $<10 \mu \mathrm{m}$. During metamorphic climax, best frequencies significantly increase, and sensitivity to lower-frequency $(<25 \mathrm{~Hz})$ stimuli tends to decline. These findings suggest that continued sensitivity to particle motion may compensate for the considerable loss of sensitivity to pressure waves observed during the developmental deaf period. Transport of a lipophilic dye from peripheral end organs to the dorsal medulla shows that fibers from the saccule in the inner ear and from the anterior lateral line both terminate in the medial vestibular nucleus. Saccular projections remain stable across larval development, whereas lateral line projections degenerate during metamorphic climax. Sensitivity to particle motion may be based on multimodal input early in development and on saccular input alone during the transition to amphibious life.
\end{abstract}

Key words: saccule; lateral line; auditory; vestibular; plasticity; development; metamorphosis

\section{Introduction}

Sensory systems develop at different rates depending on a constellation of intrinsic and extrinsic factors. Metamorphosing amphibians in particular are subject to numerous constraints affecting rate and fitness of maturation and hence are excellent models of developmental plasticity. In ranid frogs, the metamorphic transition from water to land involves considerable reorganization of both structure and function as the animal adapts to a new ecological niche. This reorganization is readily apparent in auditory pathways (Simmons and Horowitz, 2006). As aquatic animals, tadpoles are exposed to mechanical disturbances in the surrounding water that may be transduced by the auditory system as near-field particle motion or far-field pressure waves, by the vestibular system as acceleration or particle motion, or by the lateral line system as hydrodynamic flow across the body surface. In postmetamorphic amphibious frogs, lateral line function has been lost, vestibular end organs detect substrate vibrations, and the auditory system is adapted to detect propagating pressure waves in air and water. These multiple sensory modalities are not easily distinguished by the nature of the source, but they all carry biologically relevant information (Braun et al., 2002).

Bullfrog tadpoles detect pressure waves transmitted across the air-water interface, but this sensitivity changes dramatically

Received Sept. 25, 2006; revised Dec. 13, 2006; accepted Dec. 14, 2006.

This research was supported by National Institutes of Health Grant DC005257. We thank A. Alers-Fuentes, H. Baqaen, R. Dalal, R. R. Fay, E. Mullen, J. A. Simmons, and A. M. Two for assistance.

Correspondence should be addressed to Andrea M. Simmons, Department of Psychology, Box 1853, Brown University, Providence, RI 02912. E-mail: andrea_simmons@brown.edu.

DOI:10.1523/JNEUROSCI.4188-06.2007

Copyright $\odot 2007$ Society for Neuroscience $\quad$ 0270-6474/07/270782-09\$15.00/0 across metamorphosis. During a brief period of development immediately before metamorphic climax, neural activity from the auditory midbrain (torus semicircularis) exhibits a marked decline in sensitivity to pressure compared with that observed in either earlier or later larval stages (Boatright-Horowitz and Simmons, 1997). This transient period, called the deaf period, is concomitant with a temporary blockade of the oval window in the otic capsule as the opercularis low-frequency sound transduction pathway develops (Hetherington, 1987) and with a disruption of connectivity between the torus and the superior olivary nucleus (Boatright-Horowitz and Simmons, 1997; Horowitz et al., 2007). During metamorphic climax, when the tadpole becomes quadrupedal and is preparing for an amphibious lifestyle, both pressure sensitivity and midbrain-olivary connectivity are restored. Although the adaptive consequences to the animal of this transitory loss in sensitivity to pressure waves are unclear, the rapidity of this drastic reorganization argues for a tightly coupled system able to prevent a serious negative impact on survival during a period of reduced sensory sensitivity.

One way of overcoming this sensory node would be reliance on stability or increased sensitivity of other sensory components to "fill in the gap." Near-field particle motion, if detected by different neural pathways, might compensate for or even override the loss of far-field pressure sensitivity during the deaf period. Here we test this hypothesis by recording neural responses to particle motion over metamorphic development. We find that sensitivity to particle motion varies across development but remains stable during the deaf period for pressure stimulation, implying that particle and pressure sensitivity exhibit different developmental trajectories. Our data suggest that multimodal input from both the saccule and the lateral line contributes to 
particle motion sensitivity before metamorphic climax but that the saccule takes over this function during the final transition to amphibious life.

\section{Materials and Methods}

Animals. Rana catesbeiana tadpoles $(n=56)$ were obtained from a commercial supplier (Dozier Lester, Duson, LA). They were staged based on external morphological characteristics according to the Gosner staging system (Gosner, 1960) and classified into groups as follows: hatchlings (stages 21-25, the earliest freely living postembryonic animals, no hindlimb buds); early larval stages (stages 26-30, undifferentiated hindlimb buds); late larval stages (stages 31-37, progressive differentiation of hindlimbs); deaf period (stages 38-41, final hindlimb differentiation and internal forelimb differentiation); and metamorphic climax (stages $42-$ 46 , fully developed hindlimbs, emergence and full development of forelimbs, tail resorption, head and mouth transformation). Tadpoles were housed in plastic aquaria containing dechlorinated aerated water and were fed cooked unsalted spinach. The colony room was maintained on a $12 \mathrm{~h} \mathrm{light/dark} \mathrm{cycle} \mathrm{at} \mathrm{temperatures} \mathrm{ranging} \mathrm{from} 25$ to $28^{\circ} \mathrm{C}$. Experiments were conducted between the months of April and September to minimize any seasonal effects on neural responding, as observed in the midbrain of adult Rana (Goense and Feng, 2005). Animal procedures used in these experiments are consistent with guidelines established by the National Institutes of Health and were approved by the Brown University Institutional Animal Care and Use Committee.

Surgical procedures for electrophysiology. Animals were anesthetized for surgery by immersion in $0.1 \%(\mathrm{w} / \mathrm{v}) 3$-aminobenzoic acid ethyl ester methanesulfonate (MS-222), pH 7.0 (Sigma, St. Louis, MO) for 1-3 min or until reflexes disappeared. The animal was wrapped in moist gauze to facilitate cutaneous respiration. The medulla was exposed by a dorsal incision through the cartilaginous skull, and the choroid was cleared. The wound was covered with sterile Gelfoam (Upjohn, Kalamazoo, MI) moistened with $0.9 \%$ sterile saline, and the animal was placed in shallow aerated water and monitored until normal swimming behavior resumed (1-2 h). For recording, animals were immobilized with an intramuscular injection of $0.06 \%$ (w/v) D-tubocurarine chloride $(0.010 \mathrm{ml} / \mathrm{g}$; Sigma) into the tail region. Local anesthetic was swabbed on the proximal end of the tail, and a metal hook was inserted here to serve as a ground electrode during recording.

Stimulus control and calibration. We modified a shaker table system designed by Fay (1984) and used in studies of fish hearing (Fay and Edds-Walton, 1997; Lu et al., 2003) to simulate underwater particle motion in three-dimensional space. The system consists of a shallow cylindrical aluminum dish $(18 \mathrm{~cm}$ diameter, wall thickness of $1.4 \mathrm{~cm}$, depth of $7.6 \mathrm{~cm}$ ) connected by brass rods to four Brüel \& Kjær (Naerum, Denmark) Type 4810 mini-shakers for motion in the $x$ and $y$ planes and one Brüel \& Kjær Type 4809 vibration exciter for motion in the $z$ plane (from below). The mini-shakers are mounted on an aluminum platform to form a square in the horizontal plane, so that opposing pairs of shakers drive the sides of the dish in the $x$ and $y$ dimensions. When the dish is filled with water, the motion produced by the entire system simulates underwater particle motion. The shaker system is seated on two Bench Top isolation tables (Newport, Irvine CA), supported in turn by a platform made of alternating layers of bricks, wood, and neoprene mats. An aluminum rail supporting a micromanipulator (Narishige, East Meadow, NY) and the head stage of the microdrive (Trent Wells Mark 3, Coulterville, CA) is attached to the top vibration table. The entire setup is located within a single-walled sound-attenuating booth (Industrial Acoustics, Bronx, NY).

To excite the shakers at any given frequency, three separate sinusoidal signals are produced digitally by a hardware and software system [Tucker-Davis Technologies (TDT) (Alachua, FL) System 3, four channels of analog-to-digital; $10 \mathrm{kHz}$ sampling rate for signal generation]. One of these signals drives the $x$-axis pair of shakers, with $0^{\circ}$ phase to one shaker and $180^{\circ}$ to the other for reciprocal motion of the dish. The second signal drives the $y$-axis pair of shakers, again with $0^{\circ}$ phase to one shaker and $180^{\circ}$ to the other. The third signal drives the single $z$-axis shaker. The stimulation is controlled by a TDT 16-bit digital-to-analog converter

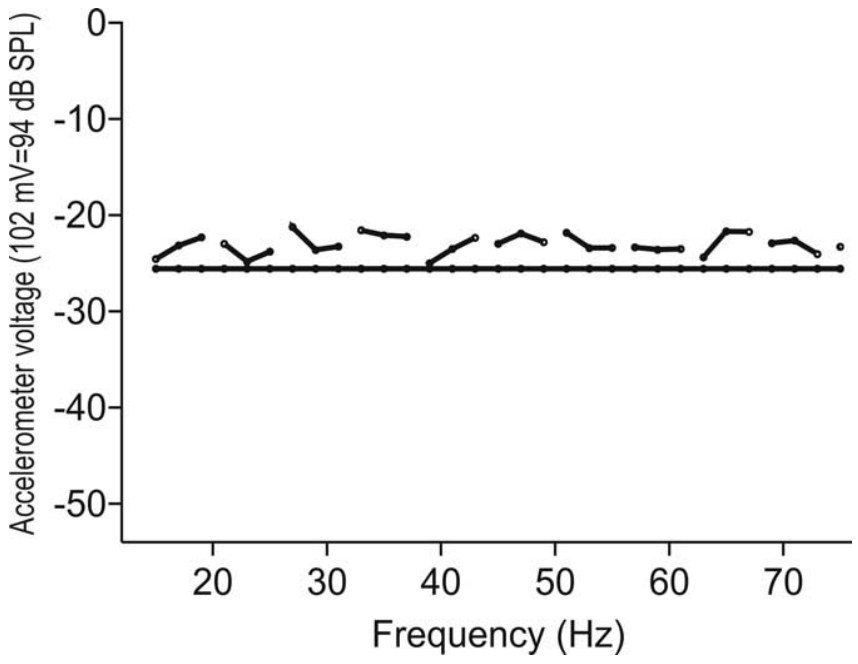

Figure 1. Sample calibration curve for $z$-axis acceleration based on input from the accelerometers $\left(102 \mathrm{mV} / \mathrm{m} / \mathrm{s}^{2}\right)$ expressed in decibels of $\mathrm{dB}$ attenuation $(94 \mathrm{~dB}$ sound pressure level $=$ $102 \mathrm{mV}$ rms). Solid horizontal line represents voltage reference level $(-25.6 \mathrm{~dB})$. The system is essentially flat ( $\pm 4 \mathrm{~dB}$ ) across the frequency range of $15-65 \mathrm{~Hz}$.

(RP2.1) and a Dell P4 computer (Dell Computer Company, Round Rock, TX). Signals from the RP2.1 converter are attenuated by TDT PA5 programmable attenuators, amplified by Crown D-75 2-channel amplifiers, and finally attenuated by $-25 \mathrm{~dB}$ by means of a custom-made attenuator connected directly to the shakers.

The actual motion of the tank in the $x, y$, and $z$ dimensions is monitored by a set of three accelerometers (model 333B50; PCB Piezotronics, Depew, NY) that supply the computer with simultaneous measurements for calibration of stimuli. The relative amplitudes and phases of the three driving signals are varied with software to produce the desired displacement or acceleration of the dish at the accelerometers. The accelerometers are attached to the dish at the boundary between the dish and the brass screws. A PCB Piezotronics model 482A22 ICP Sensor Signal Conditioner supplies the power and monitors the output of the accelerometers. Acceleration data are passed to a custom-built amplifier, low-pass filtered, and passed to the TDT RP2.1 Real-Time Processor.

Calibrations were performed by recording the output voltage of each axis at different frequency and voltage combinations. Custom-written Matlab scripts (version 6.5; MathWorks, Natick, MA) calculated the displacement and acceleration of the water particles within the circular dish based on the frequency and nominal voltage of the driving stimuli. The dish responds in a fairly linear way over the frequency range of $15-65 \mathrm{~Hz}$ (Fig. 1). In most experiments, stimulation was kept within this range. Inter-axis baseline calibrations were done before every experiment to ensure that the shakers on the different axes were receiving the same driving signal given the same voltage input by the user. There is little crosstalk between the $x$ - and $y$-axes, but stimulation at displacement levels more than $\sim 12 \mu \mathrm{m}$ along the $z$ dimension produces considerable frequency-dependent distortion along the $x$ - and $y$-axes. Crosstalk is minimal or absent at lower stimulation levels. The noise floor of the entire shaker system is $0.0225 \mu \mathrm{m}$. At the frequencies we use, which have wavelengths considerably longer than the diameter of the dish, propagating pressure waves resulting from $z$-axis displacement cannot be measured.

Recording procedures. The animal was submerged in room temperature water, except for the surgical opening, on a custom-built Lucite platform rigidly attached to the recording dish. The recording booth was maintained at a temperature of $23-25^{\circ} \mathrm{C}$ for all experiments and for all developmental stages. Tungsten microelectrodes (impedance of $<10$ $\mathrm{M} \Omega$; MicroProbe, Carlsbad, CA) were used to record neural activity from the dorsal medulla. Neural signals were monitored in real time as dot rasters, poststimulus time histograms, and spike counts and digitally stored for subsequent processing (TDT System 3). Sinusoidal stimuli $(15-65 \mathrm{~Hz} ; 10$ rise-fall cycles) were presented at a duty cycle of $12 \mathrm{~s}(4 \mathrm{~s}$ 
on, $8 \mathrm{~s}$ off) for 10-32 sweeps. Stimulus frequency and amplitude were varied to define best frequency (BF) and threshold of response. Results of extensive preliminary experiments indicated that $z$-axis stimulation produced the most robust and the most commonly encountered responses, so we focused on gathering data in response to this axis of stimulation and only tested responses to $x$ - and $y$-axes if time permitted or if $z$-axis responses could not be elicited. $z$-Axis stimulation in the shaker system provides stimulation most similar to substrate vibrations as produced in experiments with adult bullfrogs (Koyama et al., 1982). One responsive site per animal was marked with an electrolytic lesion [Stoelting (Wood Dale, IL) model 51413 constant current source; $1.5 \mu$ A of positive current for $2 \mathrm{~min}$ ]. No more than four passes (two per brain side) were attempted per animal. Experimental sessions lasted no longer than $1 \mathrm{~h}$, to avoid deterioration of the preparation.

Determination of lesion sites. At the end of recording sessions, the animal was terminally anesthetized in $0.6 \%$ MS-222 $(20 \mathrm{~min})$ and transcardially perfused with heparinized $0.9 \%(\mathrm{w} / \mathrm{v})$ saline and $4.0 \%(\mathrm{w} / \mathrm{v})$ paraformaldehyde, $\mathrm{pH}$ 7.4. The head was postfixed in paraformaldehyde for $48 \mathrm{~h}$ at $4^{\circ} \mathrm{C}$. The brain was removed, cleaned of all meninges, embedded in 5\% (w/v) agarose (ISC Bioexpress, Kaysville, UT), and sliced coronally at a thickness of $50 \mu \mathrm{m}$ on a vibratome (1000 Plus; Pelco, Redding, CA). Sections were placed on gelatin-subbed slides, stained with cresyl violet acetate $[0.5 \%(\mathrm{w} / \mathrm{v}), \mathrm{pH} 3.7$, using glacial acetic acid], and coverslipped using Cytoseal 60 acrylic mounting medium (VWR, West Chester, PA). They were visualized and imaged using an Olympus Optical (Melville, NY) BX-60 microscope connected to a MagnaFire digital camera (Optronics, Goleta, CA) and Dell computer (IEEE-1394 PCI frame grabber) and saved as 24-bit red-green-blue (RGB) TIFF files. Lesion sites were distinguished based on electrode track marks terminating in a cleared circle and surrounded by distorted cells. Their locations were identified based on atlases of the tadpole brain developed in this laboratory (Horowitz et al., 2007) (our unpublished observations).

Analysis of neural responses. Physiological responses were analyzed offline using TDT Open Explorer and Brainware (Steinhausen, Switzerland) software and custom-written Matlab scripts. Analyses included raster plots, poststimulus time histograms, spike counts, period histograms, and calculation of vector strength (VS) for quantification of phase locking to the stimulus waveform. The Rayleigh's test (Batschelet, 1981) was used to test the statistical significance of VS. To be defined as phase locked, responses had to have more than two spikes within the stimulus period per sweep across stimulus presentations and a Rayleigh's statistic $>12.8(p<0.001)$. Thresholds of response are expressed as displacement values in micrometers rather than as acceleration values (centimeters per second squared), because the latter are dependent on frequency. Statistical analyses were performed with SPSS 15 (SPSS, Chicago, IL), and graphs were plotted using SigmaPlot 8.0 (Jandel, Point Richmond, CA).

Trichrome staining. Five animals (stages $25,29,37,41$, and 44 ) were killed by immersion in $0.6 \%(\mathrm{w} / \mathrm{v})$ MS-222 for $20 \mathrm{~min}$ and then perfused as described above. Heads were removed, postfixed, embedded in paraffin, and sectioned in the coronal plane at $10 \mu \mathrm{m}$. Sections were stained with Gomori's trichrome stain (Putt, 1972). Images from a portion of this material have been presented previously (Horowitz et al., 2001).

1,1'-Dioctadecyl-5,5'-diphenyl-3,3,3',3'-tetramethylindocarbocyanine chloride labeling. Adult ranids have multiple inner ear organs that mediate sound detection, including the amphibian and basilar papillae, saccule, and lagena (Lewis et al., 1982). All of these organs are innervated by the eighth cranial nerve (nVIII). Although terminations of these separate inner ear organs in the dorsal medulla have been described for adult animals (Will and Fritzsch, 1988; McCormick, 1999), data from larval ranids have not been reported. Peripheral neuromasts in tadpoles project to the anterior lateral line nucleus (LLa) and the lateral line neuropil (LLnp) in the dorsal medulla via the anterior lateral line nerve (Fritzsch et al., 1984). To determine projections from peripheral end organs to the dorsal medulla in bullfrog tadpoles, we pressure injected the lipophilic carbocyanine dye 1,1'-dioctadecyl-5, '5' -diphenyl-3,3,3',3'-tetramethylindocarbocyanine chloride (DiI-Ph) (D7779; Invitrogen, Carlsbad, CA) into branches of nVIII innervating the saccule or lagena or into the anterior lateral line nerve of 25 animals. Lipophilic carbocyanine dyes are transported in the neuronal lipid bilayer and travel bidirectionally
(Godement et al., 1987). In addition, these dyes travel in fixed tissue and thus can be used for tracing connections from areas, such as nVIII branches, that are difficult to access in living tadpoles. Animals were killed and perfused, and the head was postfixed as described above. The medulla, anterior lateral line nerve, nVIII, and otic capsule were exposed by a ventral approach through the roof of the mouth. The saccular otolith itself was washed away with $0.9 \%$ sterile saline to allow visualization within the otic capsule of individual nVIII branches, as well as of the saccule and the lagena. DiI-Ph oil was loaded into glass microelectrodes (20-40 $\mu \mathrm{m}$ tip) held by a Narishige (Tokyo, Japan) micromanipulator attached to a manual microinjector (Sutter Instruments, Novato, CA). The electrode was lowered into the saccular $(n=15)$ or lagenar $(n=5)$ branch of nVIII or into the anterior lateral line nerve $(n=5)$. Approximately $0.10 \mu \mathrm{l}$ of dye was pressure injected. The opening was flushed with sterile saline and plugged with moistened Gelfoam. The head was reimmersed in paraformaldehyde and placed in the dark at room temperature for 3-4 weeks for travel (timing established in preliminary experiments and by Horowitz et al., 2007). After this time, the brain with the attached nerves was removed, cleared of membranes, embedded in 5\% agarose, and sliced coronally at a thickness of $50 \mu \mathrm{m}$. Sections were mounted using AquaMount (Polysciences, Warrington, PA) for visualization of fluorescence.

Imaging. Trichrome- or cresyl violet-stained sections were visualized in bright field, imaged, and saved as 24-bit RGB files. For publication, images were contrast and brightness adjusted using Photopaint (version 12; Corel, Ottawa, Ontario, Canada). DiI-Ph sections were visualized with custom-made fluorescence cubes, acquired as 24-bit RGB files, and split into separate color component grayscale files. The red channel was contrast adjusted (Photopaint), and custom wavelet deconvolution filters (Registax, version 3) were applied to highlight fiber, puncta, and cell body structures. The images were subsequently pseudocolored to red to match normal DiI-Ph and stored as 24-bit RGB images for publication.

\section{Results}

\section{Electrophysiological responses}

Single-unit $(n=9)$ and multiple-unit $(n=15)$ responses were recorded from 24 sites in 24 animals ranging from Gosner stages 30 to 45 (late larval, $n=8$; deaf period, $n=8$; metamorphic climax, $n=8$ ). All of these 24 recording sites responded robustly to $z$-axis stimulation, with little or no response to either $x$ - or $y$-axis stimulation, with the parameters we used. Active recording sites were located medially in the dorsal medulla, in a region of densely packed small cells bordering and extending in a crescent shape some distance away from the fourth ventricle (Fig. 2). In adult frogs, this cell-dense region has been subdivided and given numerous names by various investigators (Will and Fritzsch, 1988; Christensen-Dalsgaard and Walkowiak, 1999; McCormick, 1999). Because of difficulties in translating boundaries from adult to larval brains with the limited anatomical and physiological data currently available, we label this area, bounded medially by the fourth ventricle, laterally by the LLnp, and ventrally by the LLa, as the medial vestibular nucleus (MVN), consistent with the terminology used by Matesz (1979), Montgomery (1988), and Birinyi et al. (2001) in adult Rana.

Representative tuning curves from the MVN to $z$-axis stimulation from animals at different developmental stages are shown in Figure $3 a$. In general, tuning curves recorded from early and late larval (stages 30-37) and deaf period (stages 38-41) animals showed broad sensitivity over the frequency range sampled. In contrast, tuning curves from animals in metamorphic climax (stages 42-46) typically had higher thresholds at the lowfrequency $(<25 \mathrm{~Hz})$ end and also responded more robustly to higher-frequency $(>45 \mathrm{~Hz})$ stimuli. BFs for larval (early and late combined) animals ranged from 18.5 to $45.3 \mathrm{~Hz}$, with displacement thresholds at BF of 1.03-8.89 $\mu \mathrm{m}$. BFs for deaf period animals ranged from 18.5 to $55.9 \mathrm{~Hz}$, with thresholds of $0.48-$ 

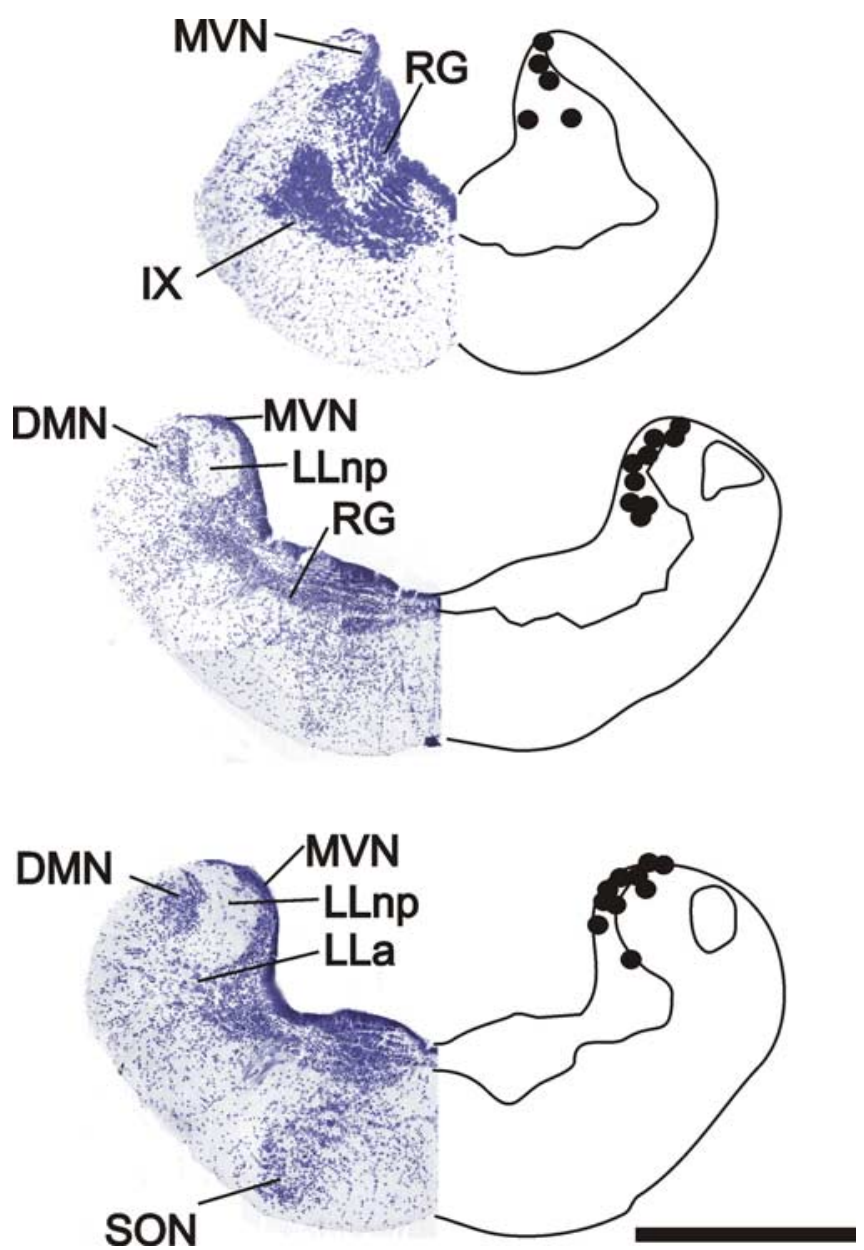

Figure 2. Schematics of the medulla (caudal to rostral, from top to bottom) based on sections from a stage 36 tadpole. Left side of each schematic shows cresyl violet-stained material, and the right side shows lesion sites (black circles, reflected to the right for clarity). Nuclear boundaries are based on published materials and our own in-house atlases. IX, Ninth cranial nerve nucleus; RG, reticular gray; SON, superior olivary nucleus. Scale bar, $500 \mu \mathrm{m}$.

$9.61 \mu \mathrm{m}$. In contrast, animals in metamorphic climax showed a more limited range of BFs, from 36.6 to $55 \mathrm{~Hz}$, with thresholds ranging from 0.55 to $6.24 \mu \mathrm{m}$ (Fig. 36 ). One-way ANOVA demonstrated a significant difference in BF based on developmental group $\left(F_{(2,23)}=4.86 ; p=0.018\right)$, with Tukey's honestly significant difference (HSD) post hoc test confirming a significant difference between larval and climax groups $(p=0.014)$. Displacement threshold at BF tended to decrease across development (Fig. $3 c$ ); however, this was not statistically significant by group ( $p=$ 0.23 ) nor was it correlated with stage (Pearson correlation coefficient, $-0.251 ; p=0.216$ ). Statistical analyses on thresholds to the highest frequency to which a particular site was responsive indicated that these thresholds were significantly lower in metamorphic climax than in either larval or deaf period groups (Tukey's HSD test, $p<0.05$ for both comparisons). There were no significant differences in thresholds across groups to the lowest frequency to which a response could be evoked.

Representative period histograms in response to suprathreshold ( $<10 \mu \mathrm{m}$ displacement) low-frequency $(\sim 20 \mathrm{~Hz})$, middlefrequency $(\sim 35 \mathrm{~Hz})$, and high-frequency $(\sim 45 \mathrm{~Hz})$ stimuli from representative animals in larval, deaf period, and climax groups are shown in Figure 4. These examples were chosen to show differences in phase-locked responses from sites with similar BFs. Histograms from the late larval (Fig. $4 a-c$, multiple-unit record- ing, BF of $40.6 \mathrm{~Hz}$ ) and deaf period (Fig. $4 d-f$, single-unit recording, $\mathrm{BF}$ of $46.1 \mathrm{~Hz}$ ) animals show significant phase locking to all stimulus frequencies within the intensity ranges studied, whereas phase locking to low-frequency stimuli in the metamorphic climax animal (Fig. 4g, multiple-unit recording, BF 45 of $\mathrm{Hz}$ ) was not statistically significant. Phase locking to higher-frequency stimulation (Fig. $4 h, i$ ) was robust in this animal. Over the entire dataset, responses from larval animals showed significant phase locking to suprathreshold stimulation over the frequency range of $15-60 \mathrm{~Hz}$; in the deaf period, significant phase locking was seen over the range of $17.5-55.1 \mathrm{~Hz}$ and in climax from 30 to $55 \mathrm{~Hz}$.

Best synchronized frequency (BSF) (the stimulus frequency at which VS is highest, at stimulus levels $<10 \mu \mathrm{m}$ displacement) ranged from 18.5 to $49.3 \mathrm{~Hz}$ in larval animals, from 18.5 to 55.1 $\mathrm{Hz}$ in deaf period animals, and from 30 to $55 \mathrm{~Hz}$ in climax animals (Fig. 5). The results of one-way ANOVA show that the difference in BSF by development group only approaches significance $\left(F_{(2,23)}=2.63 ; p=0.095\right)$. There is a significant positive correlation between BSF and developmental stage (adjusted $r^{2}=$ $0.26 ; p=0.01 ; y=-25.36+1.63 x)$. Displacement levels at BSF range between 1.91 and $9.6 \mu \mathrm{m}$, and there is no significant relationship between BSF and displacement or between displacement at BSF and stage.

Subdividing the data into single-unit and multiple-unit responses revealed some differences in response characteristics, although these differences may be confounded with developmental stage. Of the nine single-unit sites, five were recorded in larval animals, three in deaf period animals, and only one in climax. Comparing all data, regardless of developmental stage, singleunit sites showed lower BFs than multiple-unit sites (one-tailed $t$ test assuming equal variances, $t_{(22)}=2.09 ; p=0.02$ ). The lowest BFs $(\sim 18 \mathrm{~Hz})$ were obtained from single-unit responses (observed in animals at stages 32,34 , and 36), whereas the highest BFs ( $50 \mathrm{~Hz}$ and above, observed in stages 39, 42, and 43 animals) were obtained from multiple-unit recordings. Single-unit sites also had significantly higher thresholds than multiple-unit sites (mean of $5.17 \mu \mathrm{m}$ compared with $2.85 \mu \mathrm{m} ; t_{(22)}=2.11 ; p=0.02$ ) when averaged across stage. Tuning curve shapes within a developmental stage group did not differ between single- and multiple-unit sites (Fig. 3a). There was no statistical difference in the frequency range of phase locking in single- compared with multiple-unit recordings and no significant difference in BSF.

We collected data from two additional multiple-unit recording sites, one in the LLnp (from a stage 41 animal) and one in the LLa (from a stage 34 animal). Responses had BFs of 28 and $20 \mathrm{~Hz}$ and thresholds of 1.99 and $3.39 \mu \mathrm{m}$, respectively, to $z$-axis stimulation. The LLnp site also responded to $x$-axis stimulation but only at levels higher than those necessary to evoke responses to $z$-axis stimulation. The LLa site was not tested for sensitivity to $x$ or $y$-axis stimulation. Two multiple-unit recording sites were encountered in the dorsal medullary nucleus (DMN), the termination site of amphibian and basilar papillae fibers in the adult frog and the presumed homolog of the mammalian cochlear nucleus (McCormick, 1999). Activity at these sites was either poorly driven (low spike counts to frequencies $>40 \mathrm{~Hz}$ at displacement levels $>10 \mu \mathrm{m}$, no significant phase locking) or nonexistent to $z$-axis stimulation with the parameters we used. Both sites, however, showed considerable spontaneous activity. Data from these four recording sites are not included in the statistical analyses. In addition, responses to $x$-axis stimulation could be recorded from three multiple-unit sites in the MVN (animals at stages 36, 38, and 40 ). These responses could be evoked only by high-intensity 
stimulation, well above the threshold for $z$-axis responses. Because our experiments were restricted in duration and because responses to $x$ - and $y$-axis stimulation were weak overall, we did not test for directional sensitivity.

\section{General saccular anatomy}

Results of trichrome staining verify that the saccule is present, with a complement of hair cells, in early larval tadpoles (stage 29) (Fig. 6a), consistent with previous reports (Lewis and Li, 1973; Hertwig, 1987). By metamorphic climax, the organ has increased in size by $\sim 30 \%$ (Fig. 6b). Even in early larval stages (stage 25) (Fig. 6c), fibers from the saccule course through the anterior ramus of nVIII (which appears dorsally in coronal sections) to terminations in the dorsal medulla. These data suggest that the saccule forms anatomical connections with appropriate target sites even in early larval stages. The pattern of projections from the saccule to the dorsal medulla as shown by trichrome staining is similar across all larval stages investigated.

\section{Terminations of saccular and lateral line fibers}

Deposition of DiI-Ph into the saccular branch of nVIII in 13 of 15 animals (early and late larval, $n=5$; deaf period, $n=3$; climax, $n=5$ ) yielded sufficient travel beyond the insertion point of the nerve into the medulla to label terminal fields in the dorsal medulla. Data from these 13 experiments show highly consistent labeling patterns across the developmental range examined. The shape of the dorsal medulla changes over development, and the DMN is located more dorsally and medially in metamorphic climax than in early larval stages (Jacoby and Rubinson, 1983; Fritzsch et al., 1984). These changes in shape and position are reflected in our results, such that, in early larval animals, nVIII shows terminations in a laterally located cell group, identified as the developing DMN (Fig. 7a). Additional fibers pass over the LLnp, arcing dorsally to terminate in the dorsalmost region of the MVN.

Fewer and more scattered fibers course through the LLnp and terminate in the more ventral region of the MVN just dorsal to the LLa. No filled cells or clear terminals are observed within the LLnp itself or within the LLa. Very similar projection patterns are observed in late larval stages (data not shown), the deaf period (Fig. 7b), and metamorphic climax (Fig. 7c). In these stages, labeled fibers appear to extend more ventrally throughout the MVN than in earlier stages, resulting in heavier label and more terminations in ventral areas of the MVN. High-magnification images show numerous puncta and terminals in the MVN (Fig. $7 d$ ), particularly around cell bodies. Injections distal to the saccular branching, nearer the end organ, reliably produce label of
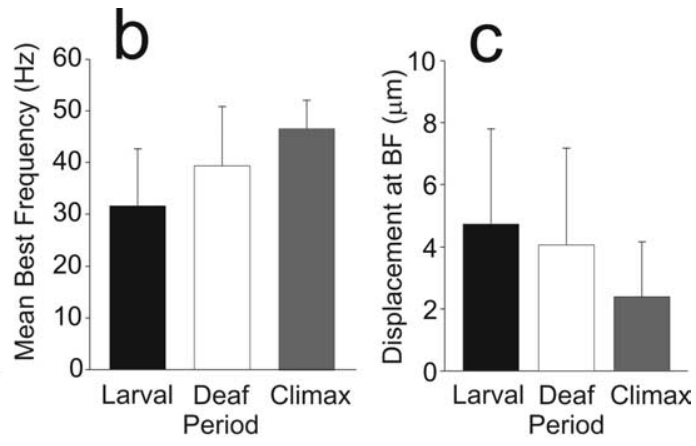

Figure 3. Developmental changes in auditory tuning. $\boldsymbol{a}$, Tuning curves from representative animals in each developmental group showing threshold in displacement (micrometers) at best frequency (hertz). Data are derived from tadpoles at stages 30 (multiple-unit recording), 36 (multiple-unit recording), 41 (single-unit recording), and 42 (multiple-unit recording). $\boldsymbol{b}$, Differthree groups are significantly different $(p<0.05)$. Post hoc tests (Tukey's HSD test) indicate that the larval and climax groups show the greatest difference. c, Changes in displacement thresholds by developmental group for the entire dataset. Although thresholds are absolutely lower in climax compared with larval groups, the difference is not statistically significant. Error bars
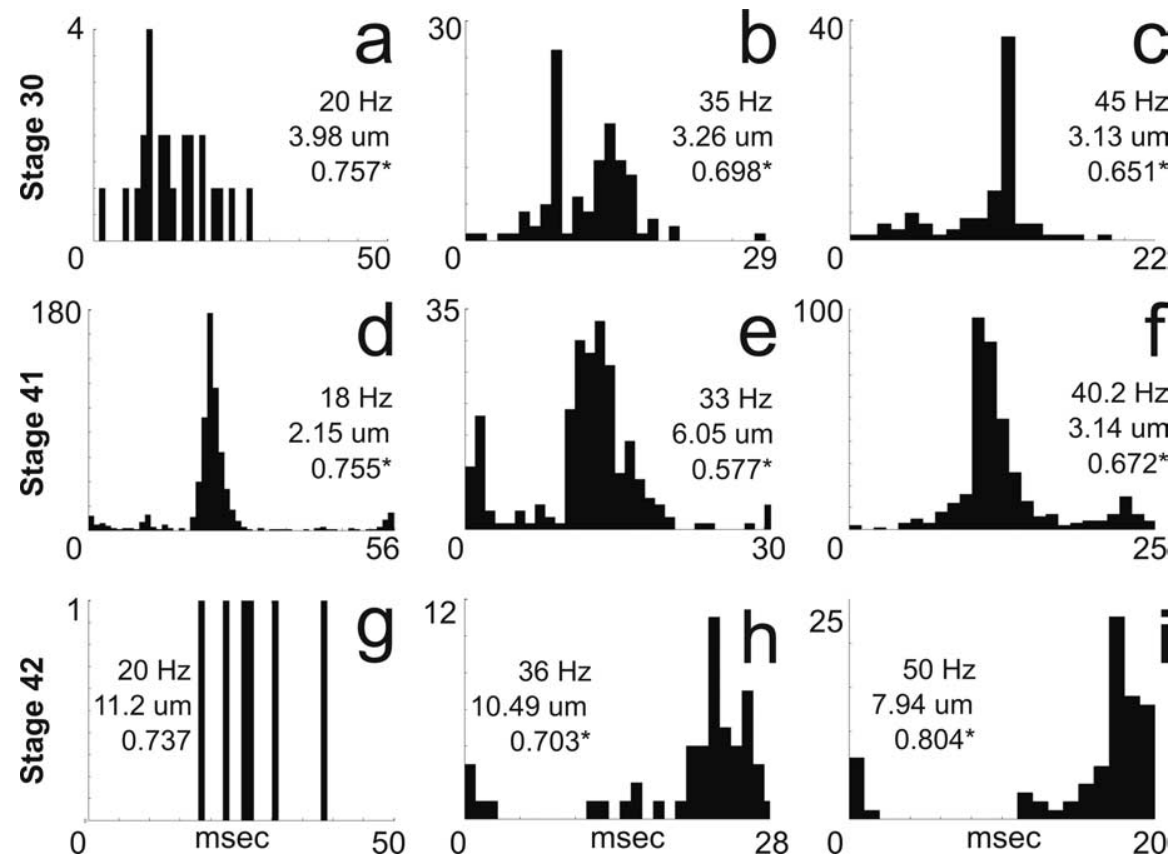

Figure 4. Period histograms from representative late larval $(\boldsymbol{a}-\boldsymbol{c}$, multiple-unit recording, $\mathrm{BF}$ of $40.6 \mathrm{~Hz}$, threshold at $\mathrm{BF}$ of $1.52 \mu \mathrm{m})$, deaf period ( $\boldsymbol{d}-\boldsymbol{f}$, single-unit recording, BF of $46.1 \mathrm{~Hz}$, threshold at BF of $9.6 \mu \mathrm{m})$, and climax $(\boldsymbol{g}$ - $\boldsymbol{i}$, multiple-unit high-frequency $(\boldsymbol{c}, \boldsymbol{f}, \boldsymbol{i})$ stimuli. Stimulus frequency, displacement values, and VS are listed by each histogram. Asterisk indicates significant VS based on the Rayleigh's test. Bin width for period histograms is $1 \mathrm{~ms}$. The vertical axis shows the number of spikes per bin, and the horizontal axis shows one stimulus period in milliseconds.

the end organ itself, including supporting cells and occasionally hair cells (Fig. 7e).

DiI-Ph label of the anterior lateral line nerve $(n=5)$ results in extensive fiber label from the nerve entry site toward both the MVN and the LLa (Fig. 8a), as well as labeled terminals, puncta, and cell bodies in the LLnp, the MVN (Fig. 8b), and the LLa. There was no label of the DMN, as expected (Jacoby and Rubinson, 1983; Fritzsch et al., 1984). Lateral line terminations could not be traced in metamorphic climax because the system begins to degenerate at the beginning of climax. Because the lagena responds to particle motion in adult fish (Lu et al., 2003) and to substrate vibration in adult frogs (Cortopassi and Lewis, 1998), 


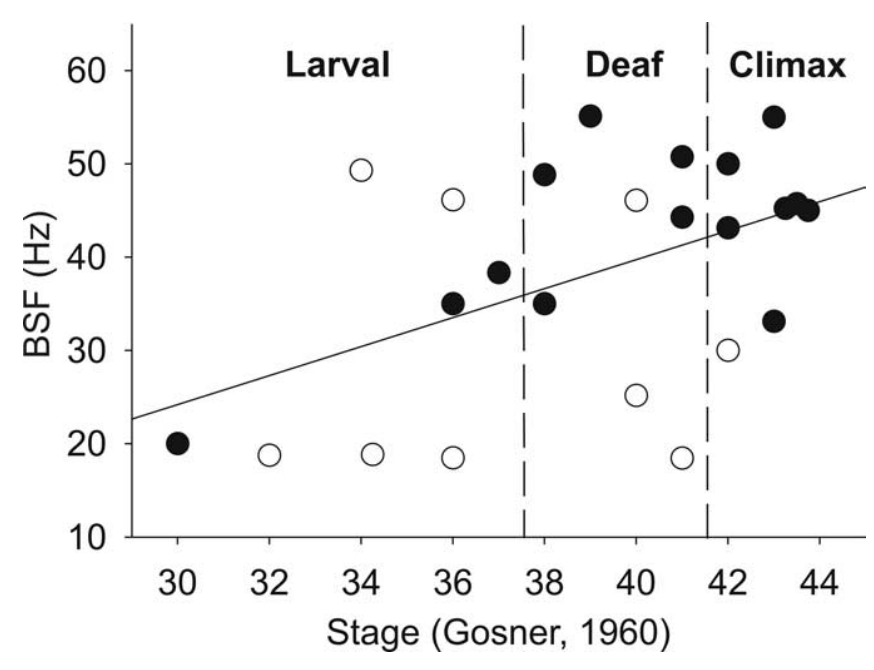

Figure 5. Changes in best synchronized frequency by Gosner stage for all animals. Best synchronized frequency is determined based on highest vector strength for any stimulus frequency that is significant based on the Rayleigh's test. Open circles show single-unit recordings, and filled circles show multiple-unit recordings. The black line is a first-order regression line $\left(r^{2}=0.259\right)$ through the entire dataset; vertical dashed lines demarcate the deaf period.

DiI-Ph deposition in the lagenar branch of $\mathrm{nVIII}(n=5)$ was also performed. The lagena showed different projection patterns from both the saccule and the lateral line nerve, with fiber label in the medial region of LLnp and in the vestibulospinal tract, and terminal and puncta label of the dorsomedial margin of the MVN, the lateral vestibular nucleus, and the medial reticular gray (data not shown).

\section{Discussion}

As developing aquatic and then amphibious animals, bullfrogs are subject to both particle and pressure components of sound. Here we show that these two components do not mature in parallel but follow different developmental trajectories across metamorphosis. In particular, a salient feature of auditory system development to pressure sensitivity, the deaf period, is not present when auditory function is indexed by responses to the particle motion component of sound. This preservation of sensitivity to particle motion during the pressure-based deaf period allows the animal the continued ability to detect environmental changes, such as the low-frequency water movements produced by other aquatic organisms (Bleckmann et al., 1991), during a time of considerable reorganization in both the middle ear and central auditory pathways.

Although responses to particle motion do not show a deaf period effect, cells in the MVN responsive to particle motion produced by $z$-axis (vertical) vibration of fluid surrounding the animal do differ in certain physiological characteristics across development. BFs from animals in metamorphic climax are significantly higher as well as more restricted in frequency range than those from animals in early and late larval stages, in which BFs span a wider-frequency range and especially includes low $(<25 \mathrm{~Hz})$ frequencies. Neural tuning curves from both larval and deaf period tadpoles typically are broad in shape over the frequency range we investigated. In contrast, tuning curves from tadpoles in climax are more V-shaped, with elevated thresholds to frequencies $<25 \mathrm{~Hz}$ and lower thresholds to frequencies $>45$ $\mathrm{Hz}$. Displacement thresholds at BF tend to remain stable across development, albeit with a trend toward lower thresholds in metamorphic climax. Thresholds to high frequencies (both at
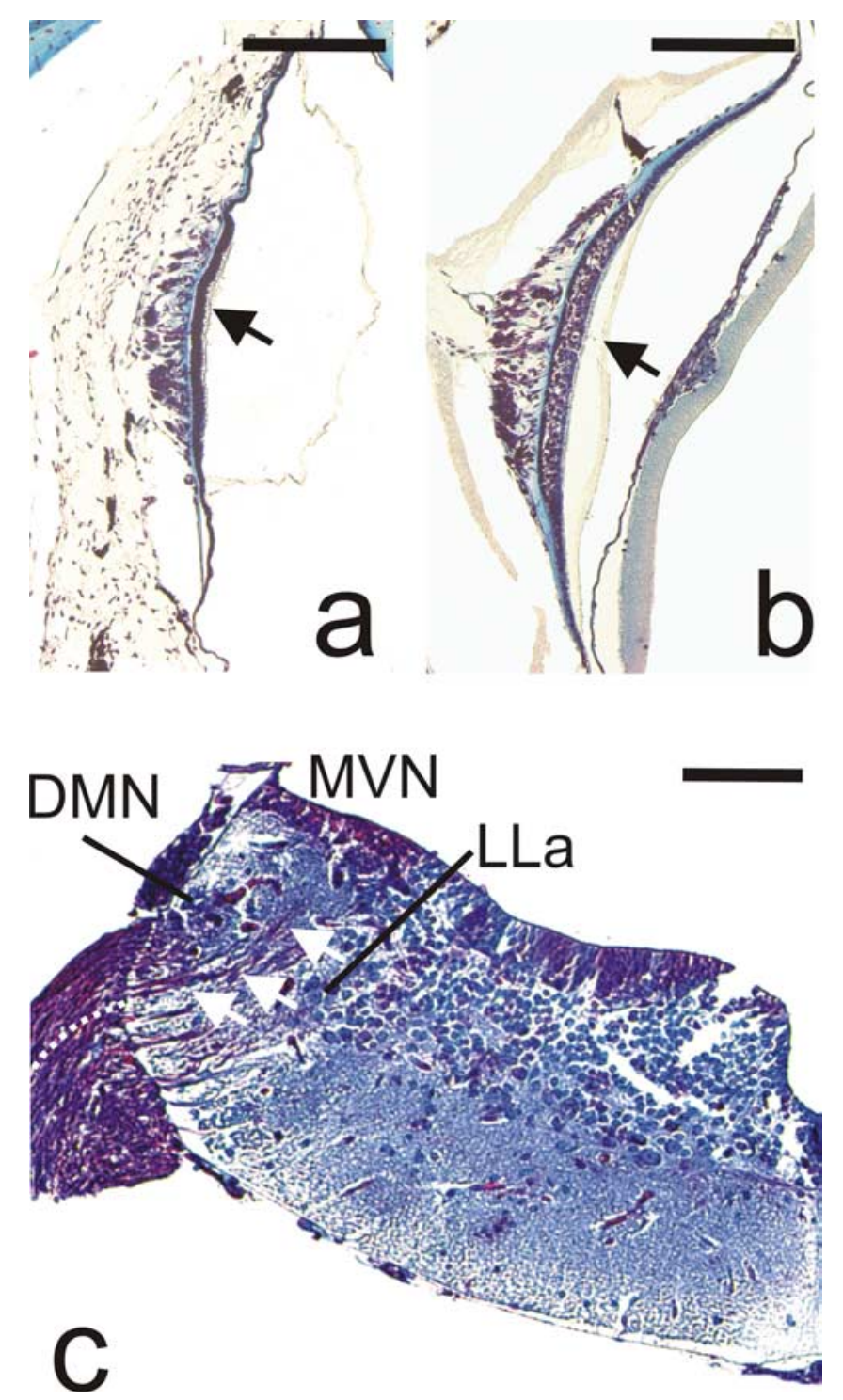

Figure 6. Trichrome staining of saccule and medulla. Trichrome-stained coronal sections, 10 $\mu \mathrm{m}$ thick, of the inner ear of stage $29(\boldsymbol{a})$ and stage $44(\boldsymbol{b})$ tadpole showing the saccule. Black arrows indicate the hair-cell layer of the sensory macula. Lateral is to the right, and dorsal is up. c, Coronal section, $10 \mu \mathrm{m}$ thick, of the medulla of a stage 25 bullfrog tadpole at the level of the insertion of the anterior (saccular) branch of nVIII. Fibers above the white dotted line are typically saccular. White arrows indicate saccular fibers traversing the medulla toward the DMN and MVN but dorsal to the LLa. Higher magnification shows terminations in and near the MVN region. Scale bars: $\boldsymbol{a}, \boldsymbol{b}, 200 \mu \mathrm{m} ; \boldsymbol{c}, 100 \mu \mathrm{m}$.

and around BF) are significantly lower in metamorphic climax than in larval stages, however. Consistent with the stage differences in BF, phase-locked neural responses are present to a wider frequency range of stimulation in larval and deaf period animals than in climax animals, and BSFs tend to be lower in larval than in climax animals. Restriction of the range of significant phase locking is also observed in response to acoustic pressure waves across a similar developmental range (Boatright-Horowitz et al., 1999). Cells in the auditory midbrain of early larval tadpoles show significant phase locking to noise bursts with modulation rates of up to $250 \mathrm{~Hz}$, whereas phase locking in climax is restricted to rates $<100 \mathrm{~Hz}$. This suggests that phase locking to a broad range of temporal features of acoustic stimuli is a marker of particularly plastic developmental periods across different modalities.

BFs to $z$-axis particle motion in tadpoles are within the range 

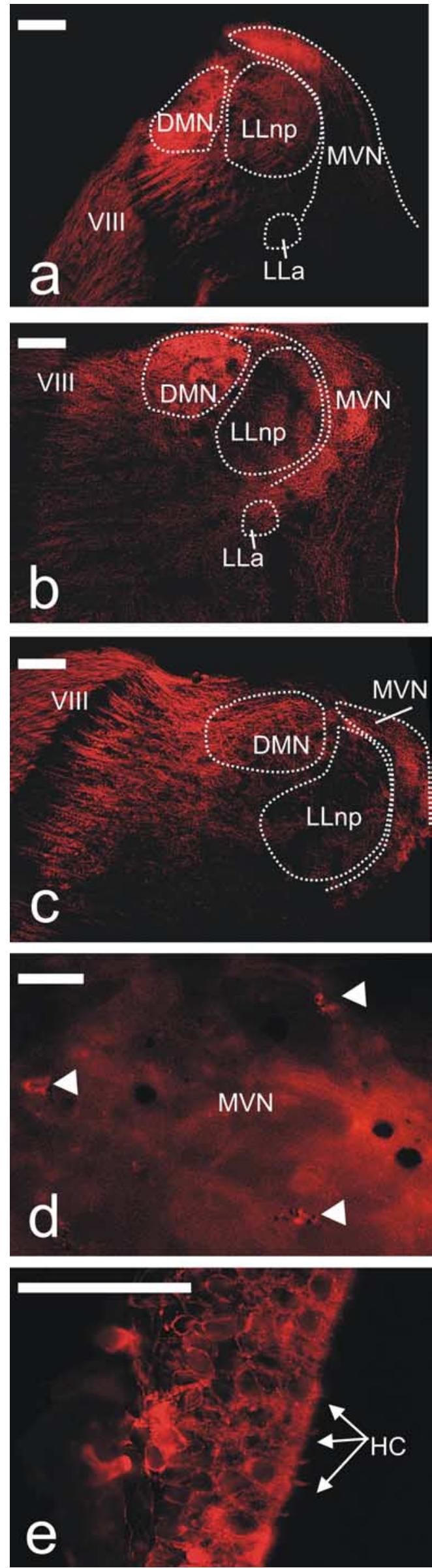
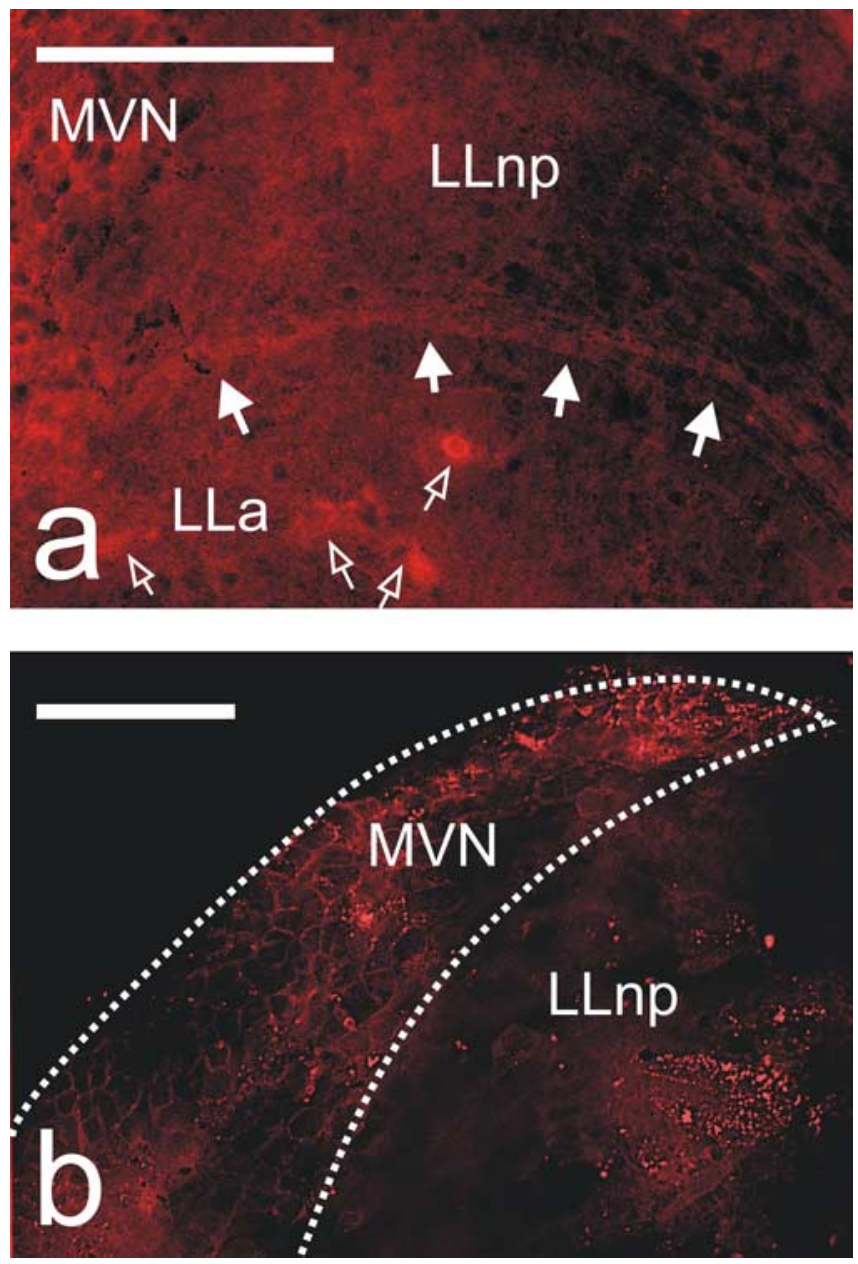

Figure 8. Label of the dorsal medulla resulting from Dil-Ph deposition in the anterior lateral line nerve of a stage 30 tadpole. $\boldsymbol{a}$, Filled white arrows indicate the course of labeled fibers from the entry of nVIII (to the right; data not shown) through the LLnp. Open arrows indicate filled cells in and around the LLa. $\boldsymbol{b}$, Higher-power image showing puncta and terminal label in both the MVN and LLnp. Dotted lines indicate the MVN. Lateral is to the right, and dorsal is up in all figures. Scale bars, $100 \mu \mathrm{m}$.

observed to $z$-axis substrate vibrations in adult Rana $[20-150 \mathrm{~Hz}$, nVIII (Koyama et al., 1982; Yu et al., 1991); 10-80 Hz, dorsal medulla (Christensen-Dalsgaard and Walkowiak, 1999)]. Lowest thresholds in adults, which are typically expressed in units of acceleration rather than displacement, are considerably lower $\left(0.005 \mathrm{~cm} / \mathrm{s}^{2}, \mathrm{nVIII} ; 0.01 \mathrm{~cm} / \mathrm{s}^{2}\right.$, dorsal medulla) than the lowest acceleration thresholds recorded in tadpoles $\left(1.43 \mathrm{~cm} / \mathrm{s}^{2}\right)$. This latter threshold is in turn considerably lower than the accelerations producing predator-induced hatching of egg clutches of the

\section{$\leftarrow$}

Figure 7. Medullary termination of the saccular branch of nVIII across development as shown by Dil-Ph tracing (bright red). $\boldsymbol{a}$, Fiber projection pattern from the saccular branch of nVIII in a stage 28 (early larval) tadpole. Fibers course through the DMN and dorsal MVN and, to a lesser extent, through the LLnp. Terminations are seen in the DMN and MVN but not in the LLnp or LLa. Similar patterns are observed in stage 38 (deaf period) (b) and stage 42 (metamorphic climax) (c) tadpoles. Note that the position of the DMN changes across larval development from a more lateral position in early larval animals to a more medial and dorsal position in metamorphic climax. The area of the LLnp is demarcated by the white polygon. This region becomes relatively more compressed as the DMN moves more dorsally and medially. $\boldsymbol{d}$, Terminal and puncta label in the MVN of a stage 45 tadpole. White arrowheads indicate putative terminals. $e$, Label of the saccular end organ in a stage 45 tadpole, showing label of supporting and hair cells (HC, white arrows). Scale bars: $\boldsymbol{a}-\boldsymbol{c}, 200 \mu \mathrm{m} ; \boldsymbol{d}, 10 \mu \mathrm{m} ; \boldsymbol{e}, 20 \mu \mathrm{m}$. 
red-eyed tree frog (Warkentin, 2005). These threshold differences may reflect changes in the mass and overall size of the saccule as well as in numbers of hair cells as the animal develops from embryonic to adult stages (Lewis and Li, 1973), and they point to an increased sensitivity to $z$-axis stimulation as the animal ages. It is interesting to note that, in several species of fish, as otolith mass increases, the saccule becomes more sensitive to lower-frequency stimuli with sharper frequency tuning around $\mathrm{BF}$ and that sensitivity to vestibular stimulation is higher but with slower responses (Lychakov and Rebane, 2000). This is the opposite pattern than what we observed during metamorphic development, but it is consistent with the greater low-frequency sensitivity of the adult compared with the tadpole saccule.

In all metamorphic stages, the saccule projects to both the MVN and the DMN. The MVN projections appear to be more extensive in tadpoles than in adult frogs (Birinyi et al., 2001). This suggests a developmental pruning of saccular projections during postmetamorphic life, possibly reflecting a reduction of input from underwater particle motion and an increase in input from other vestibular organs as the animal adopts an amphibious lifestyle. Saccular projections to the DMN appear to remain unchanged throughout larval life and are similar in extent to what has been observed in adult frogs (Matesz, 1979; McCormick, 1999). The DMN projections may mediate the residual highthreshold responsiveness to pressure stimulation sometimes observed during the deaf period (Boatright-Horowitz and Simmons, 1997). It has been suggested that the saccule in adult frogs operates as a mixed auditory/vestibular organ (Lewis, 1983). Our data suggest that this is also the case in developing frogs.

In this study, we did not trace projections from the semicircular canals or from the amphibian or the basilar papilla. In adult frogs (Will and Fritzsch, 1988), some papillar fibers project to the caudal nucleus, which constitutes part of the area we term the MVN in tadpoles. The amphibian papilla responds to highfrequency $(80-150 \mathrm{~Hz})$ substrate vibrations in adult bullfrogs (Yu et al., 1991). Because we did not systematically test responses to frequencies $>80 \mathrm{~Hz}$, we cannot eliminate the amphibian papilla in the coding of particle motion, but there is no evidence, even in adults, that this organ responds to the low frequencies we tested.

Interconnections of the MVN with other auditory brainstem nuclei remain essentially stable across development, in contrast with the considerable metamorphic reorganization of other pathways (Horowitz et al., 2007). The LLa, for example, shows substantial afferent and efferent connectivity with the superior olivary nucleus and the torus semicircularis during early and late larval stages, but these begin declining during the deaf period and are gone by mid-climax. Moreover, during the deaf period, a disconnection in afferent and efferent connectivity between the superior olivary nucleus and the torus appears, but these connections reemerge and become more extensive in climax. The relative stability of MVN anatomy and function points to the biological importance of maintaining good sensitivity to particle motion throughout larval life.

We interpret the functional responsiveness of the MVN, along with our anatomical findings that both the saccule and the lateral line project to this area, as showing that sensitivity to particle motion before metamorphic climax is mediated by both saccular and lateral line input. The truncation in the frequency range of sensitivity to particle motion that emerges as the animal reaches metamorphic climax occurs concomitant with the loss of connectivity between the anterior lateral line nerve to the MVN during this developmental period. Our interpretation is based on the assumption that the lateral line in bullfrog tadpoles is functional at early larval stages and that it is most sensitive to frequencies $<25 \mathrm{~Hz}$. Direct measurements of responsiveness of lateral line neuromasts or nerve fibers have not been reported in bullfrog tadpoles, but, in Xenopus laevis tadpoles, electrical activity "essentially adult in character" can be recorded from the lateral line nerve as early as Nieuwkoop-Faber stage 38 (Zimmerman, 1979), comparable with Gosner stage 21. Adult Xenopus lateral line fibers respond maximally to frequencies between 10 and $40 \mathrm{~Hz}$ (Strelioff and Honrubia, 1978), and our data predict that anterior lateral line fibers in bullfrog tadpoles will be most responsive to frequencies at the low end of this range.

The developmental changes we observed suggest a substantial amount of integration of auditory and lateral line systems at firstorder medullary nuclei. These two interacting modalities provide stable particle motion input during the deaf period of loss of pressure sensitivity. The emergence of forelimbs and reemergence of pressure sensitivity, presumably via the opercularis transduction pathway (Hetherington, 1987), mark the end of the deaf period and the onset of degeneration of the lateral line system. This suggests that developmental trajectories of the three hair-cell-mediated systems, vestibular, lateral line, and auditory, are coordinated in such a manner as to minimize loss of overall sensitivity of the octavolateralis senses while allowing major reconstruction and recalibration of sensory systems to occur.

Larsell (1934) proposed that, during metamorphic climax, lateral line-sensitive cells in the dorsal medulla become transformed into auditory-sensitive cells. This hypothesis has been attacked on the basis of data showing that lateral line and nVIII input terminate in separate medullary areas (LLnp and DMN, respectively), even in early larval stages (Jacoby and Rubinson, 1983; Fritzsch et al., 1984). We suggest that Larsell's hypothesis be reformulated to postulate a transformation during climax from bimodal lateral line/saccular sensitivity to saccular sensitivity alone. We would thus expect the anterior lateral line and the saccular branch of nVIII to terminate on the same MVN neurons. Single-unit recordings combined with iontophoresis are required to test this hypothesis.

\section{References}

Batschelet E (1981) Circular statistics in biology. New York: Academic.

Birinyi A, Straka H, Matesz C, Dieringer N (2001) Location of dye-coupled second order and of efferent vestibular neurons labeled from individual semicircular canal or otolith organs in the frog. Brain Res 921:44-59.

Bleckmann H, Breithaupt T, Blickhan R, Tautz J (1991) The time course and frequency content of hydrodynamic events caused by moving fish, frogs, and crustaceans. J Comp Physiol A Neuroethol Sens Neural Behav Physiol 168:749-757.

Boatright-Horowitz SS, Simmons AM (1997) Transient “deafness" accompanies auditory development during metamorphosis from tadpole to frog. Proc Natl Acad Sci USA 94:14877-14882.

Boatright-Horowitz SS, Garabedian CE, Odabashian KH, Simmons AM (1999) Coding of amplitude modulation in the auditory midbrain of the bullfrog (Rana catesbeiana) across metamorphosis. J Comp Physiol A Neuroethol Sens Neural Behav Physiol 184:219-231.

Braun CB, Coombs S, Fay RR (2002) What is the nature of multisensory interaction between octavolateralis sub-systems? Brain Behav Evol 59:162-176.

Christensen-Dalsgaard J, Walkowiak W (1999) In vitro and in vivo responses of saccular and caudal nucleus neurons in the grassfrog (Rana temporaria). Eur J Morphol 37:206-210.

Cortopassi KA, Lewis ER (1998) A comparison of the linear tuning properties of two classes of axons in the bullfrog lagena. Brain Behav Evol 51:331-348.

Fay RR (1984) The goldfish ear codes the axis of acoustic particle motion in three dimensions. Science 225:951-954. 
Fay RR, Edds-Walton PL (1997) Diversity in frequency response properties of saccular afferents of the toadfish, Opsanus tau. Hear Res 113:235-246.

Fritzsch B, Nikundiwe AM, Will U (1984) Projection patterns of lateral line afferents in anurans: a comparative HRP study. J Comp Neurol 229:451-469.

Godement P, Vanselow J, Thanos S, Bonhoeffer F (1987) A study in developing visual systems with a new method of staining neurones and their processes in fixed tissue. Development 101:697-713.

Goense JBM, Feng AS (2005) Seasonal changes in frequency tuning and temporal processing in single neurons in the frog auditory midbrain. J Neurobiol 65:22-36.

Gosner KL (1960) A simplified table for staging anuran embryos and larvae with notes on identification. Herpetologica 16:183-190.

Hertwig I (1987) Morphogenesis of the inner ear of Rana temporaria (Amphibia, Anura). Zoomorphology 107:103-114.

Hetherington T (1987) Timing of development of the middle ear of Anura. Zoomorphology 106:289-300.

Horowitz SS, Chapman JA, Kaya U, Simmons AM (2001) Metamorphic development of the bronchial columella of the larval bullfrog (Rana catesbeiana). Hear Res 154:12-25.

Horowitz SS, Chapman JA, Simmons AM (2007) Plasticity of auditory medullary-midbrain connectivity across metamorphic development. Brain Behav Evol 69:1-19.

Jacoby J, Rubinson K (1983) The acoustic and lateral line nuclei are distinct in the premetamorphic frog, Rana catesbeiana. J Comp Neurol 216:152-161.

Koyama H, Lewis ER, Leverenz EL, Baird RA (1982) Acute seismic sensitivity in the bullfrog ear. Brain Res 250:168-172.

Larsell O (1934) The differentiation of the peripheral and central acoustic apparatus in the frog. J Comp Neurol 60:473-527.

Lewis ER (1983) Dual acoustical sensitivity in frogs. In: Hearing: physiological bases and psychophysics (Klinke R, Hartmann R, eds), pp 61-69. New York: Springer.
Lewis ER, Li CW (1973) Evidence concerning the morphogenesis of saccular receptors in the bullfrog (Rana catesbeiana). J Morphol 139:351-362.

Lewis ER, Baird RA, Leverenz EL, Koyama H (1982) Inner ear: dye injection reveals peripheral origins of specific sensitivities. Science 215:1641-1643.

Lu Z, Xu Z, Buchser WJ (2003) Acoustic response properties of lagenar nerve fibers in the sleeper goby, Dormitator latifrons. J Comp Physiol A Neuroethol Sens Neural Behav Physiol 189:889-905.

Lychakov DV, Rebane YT (2000) Otolith regularities. Hear Res 143:83-102.

Matesz C (1979) Central projection of the VIIIth cranial nerve in the frog. Neuroscience 4:2061-2071.

Montgomery N (1988) Projections of the vestibular and cerebellar nuclei in Rana pipiens. Brain Behav Evol 31:82-95.

McCormick CA (1999) Anatomy of the central auditory pathways of fish and amphibians. In: Comparative hearing: fish and amphibians (Fay RR, Popper AN, eds), pp 155-217. New York: Springer.

Putt FA (1972) Manual of histopathological staining methods. New York: Wiley.

Simmons AM, Horowitz SS (2006) Plasticity in the auditory system across metamorphosis. In: Hearing and sound communication in amphibians (Narins PM, Feng AS, Fay RR, Popper AN, eds), pp 291-322. New York: Springer.

Strelioff D, Honrubia V (1978) Neural transduction in Xenopus laevis lateral line system. J Neurophysiol 41:432-443.

Warkentin KM (2005) How do embryos assess risk? Vibrational cues in predator-induced hatching of red-eyed treefrogs. Anim Behav 70:59-71.

Will U, Fritzsch B (1988) The eighth nerve of amphibians: peripheral and central distribution. In: The evolution of the amphibian auditory system (Fritzsch B, Ryan MJ, Wilczynski W, Hetherington TE, Walkowiak W, eds), pp 159-183. New York: Wiley.

Yu XL, Lewis ER, Feld D (1991) Seismic and auditory tuning curves from bullfrog saccular and amphibian papillar axons. J Comp Physiol A Neuroethol Sens Neural Behav Physiol 169:241-248.

Zimmerman DM (1979) Onset of neural function in the lateral line. Nature 282:82-84 